\title{
BEBIDA FUNCIONAL CON POTENCIAL ANTIDIABÉTICO Y ANTIHIPERTENSIVO ELABORADA CON MAÍZ AZUL Y FRIJOL NEGRO BIOPROCESADOS
}

\section{FUNCTIONAL BEVERAGE PRODUCED WITH BIOPROCESSED BLUE CORN AND BLACK BEANS WITH ANTIHYPERTENSIVE AND ANTIDIABETIC POTENTIAL}

\author{
Jorge Milán-Carrillo1,2, Roberto Gutiérrez-Dorado1,2, Edith O. Cuevas-Rodríguez ${ }^{1,2}$, \\ Luis M. Sánchez-Magaña ${ }^{1,2}$, Jesús J. Rochín-Medina' y Cuauhtémoc Reyes-Moreno ${ }^{1,2 *}$
}

\begin{abstract}
'Programa Regional de Posgrado en Biotecnología, Facultad de Ciencias Químico Biológicas (FCQB), Universidad Autónoma de Sinaloa (UAS), Cd. Universitaria, Blvd Las Américas S/N. 80000, Culiacán, Sinaloa, México. ${ }^{2}$ Programa de Posgrado en Ciencia y Tecnología de Alimentos, FCQB-UAS, Cd. Universitaria, Blvd. Las Américas S/N. 80000, Culiacán, Sinaloa, México.
\end{abstract}

*Autor para correspondencia (creyes@uas.edu.mx)

\section{RESUMEN}

México, centro de origen y domesticación del maíz (Zea mays L.), cuenta con la mayor diversidad de esta especie en el mundo, con aproximadamente 59 razas. Los maíces azules, ricos en antocianinas, pueden ser considerados como nutracéuticos debido al efecto preventivo de estos pigmentos contra el estrés oxidativo, enfermedades crónico-degenerativas y cáncer. De la misma manera, México es reconocido como centro de origen y diversidad de alrededor de 70 especies del género Phaseolus, de las cuales destaca por su importancia económica, social, alimenticia y cultural el frijol común ( $P$. vulgaris $\mathrm{L}$.). El frijol es una fuente buena de proteínas, es rico en fibra soluble y ácidos grasos poliinsaturados; además, contiene compuestos fenólicos con actividad antioxidante. El objetivo de esta investigación fue desarrollar una bebida funcional, con valor nutricional y potencial antihipertensivo y antidiabético alto, a partir de una mezcla 60:40 de harinas de granos integrales de maíz azul y frijol negro bioprocesadas en condiciones óptimas de fermentación en estado sólido (FES). Para la producción de harinas de maíz azul y frijol negro bioprocesados optimizadas (HMABO, HFNBO) se aplicaron temperaturas/tiempos de fermentación de $37{ }^{\circ} \mathrm{C} / 90 \mathrm{~h}$ y de $38{ }^{\circ} \mathrm{C} / 100 \mathrm{~h}$, respectivamente; estas condiciones se optimizaron en estudios previos maximizando actividad antioxidante (AAox) y compuestos fenólicos totales (CFT). El bioproceso de la FES incrementó AAox (36.8 a $75.6 \%$ ), CFT (85 a $127 \%$ ) y potencial antihipertensivo y antidiabético de maíz azul y frijol negro. Una porción de $200 \mathrm{~mL}$ de bebida funcional, preparada con $25 \mathrm{~g}$ mezcla ( $60 \%$ HMABO + $40 \%$ HFNBO), cubre $34.2 \%$ y $23.4 \%$ de requerimientos diarios de proteína para niños de 1 a 3 y de 4 a 8 años, respectivamente, y de 90 a 150 $\%$ del consumo diario recomendado de antioxidantes. Por su valor nutricional y potencial antihipertensivo y antidiabético, esta bebida funcional puede ser empleada para promover la salud y como alternativa a bebidas de contenido calórico alto y valor nutricional bajo.

Palabras clave: maíz azul, frijol negro, fermentación en estado sólido, bebida funcional.

\section{SUMMARY}

Mexico, the center of origin and domestication of maize (Zea mays L.), has the largest diversity of this species in the world, with approximately 59 races. Anthocyanin-rich blue corn can be considered as nutraceutical because of the preventive effects of these pigments against oxidative stress, chronicdegenerative diseases and cancer. Likewise, Mexico is recognized as a center of origin and diversity of about 70 species of Phaseolus, of which common bean (P. vulgaris L.) stands out for its economic, social, nutritional and cultural importance. Beans are a good source of protein, rich in soluble fiber and polyunsaturated fatty acids; in addition, beans contain phenolic compounds with antioxidant activity. This research developed a functional beverage, with high nutritional value and antihypertensive and antidiabetic potential from a 60:40 mixture of whole grains flours of blue corn and black common bean bioprocessed under optimum solid state fermentation (SSF) conditions. For production of optimized bioprocessed blue corn and black common bean flours (OBBMF, OBBCBF) temperatures/fermentation times of $37^{\circ} \mathrm{C} / 90 \mathrm{~h}$ and $38{ }^{\circ} \mathrm{C} / 100 \mathrm{~h}$, respectively, were applied. These conditions were optimized in previous studies to maximize antioxidant activity (AoxA) and total phenolic compunds (TPC). The SSF bioprocess increased AoxA (from 36.8 to 75.6 $\%$ ), TPC (from 85 to $127 \%$ ) and antihypertensive and antidiabetic potential of blue corn and black beans. A $200 \mathrm{~mL}$ serving of this functional beverage, prepared with $25 \mathrm{~g}$ mixture (60\% OBBMF + $40 \%$ OBCBF) supplies $34.2 \%$ and $23.4 \%$ of the daily protein requirements for children from ages 1 to 3 and 4 to 8 , respectively, and 90 to $150 \%$ of the recommended daily intake of antioxidants. Due to its nutritional value and antihypertensive and antidiabetic potential, this functional beverage might be used to promote health and as an alternative to beverages with a high caloric content and low nutritional value.

Index words: blue corn, black common beans, solid state fermentation, functional beverage.

\section{INTRODUCCIÓN}

En México, centro de origen, domesticación y diversificación del maíz (Zea mays L.), existen 59 razas de acuerdo con la clasificación basada en características morfológicas e isoenzimáticas, lo que representan un significativo porcentaje de las 220 a 300 razas de maíz existentes en el continente americano (Fernández et al., 2013; Sánchez et al., 2000). Todos los maíces nativos contienen compuestos fenólicos de alto valor, de modo que constituyen un reservorio y patrimonio genético por explorar. Los maíces azules, ricos en antocianinas, pueden ser considerados como nutracéuticos debido al efecto preventivo de estos pigmentos contra el estrés oxidativo, las enfermedades 
crónico-degenerativas y el cáncer. Otros importantes fitoquímicos son los fitoesteroles y policosanoles por sus probados efectos hipocolesterolémicos (Serna-Saldívar et al., 2013).

México es reconocido como centro de origen y de diversidad de muchas especies, entre las que se encuentran aproximadamente 70 del género Phaseolus, de las que destaca por su importancia económica, social, biológica, alimenticia y cultural el frijol común (P. vulgaris L.). Por su superficie sembrada, es el segundo cultivo en importancia después del maíz; además, su grano es un alimento básico que hace un notable aporte proteínico a la dieta humana, sobre todo en poblaciones rurales y urbanas de escasos recursos (Avendaño et al., 2004). El frijol es una fuente razonablemente buena de proteínas y carbohidratos complejos; es rico en fibra soluble, vitaminas hidrosolubles, minerales y ácidos grasos poliinsaturados; además, contiene compuestos fenólicos con actividad antioxidante (Chávez-Santoscoy et al., 2016).

Algunos compuestos fenólicos presentes en cereales y leguminosas ejercen actividad antidiabética a través de la inhibición de enzimas que hidrolizan carbohidratos (a-amilasa, a-glucosidasa) (Kim et al., 2005; SánchezMagaña et al., 2014). Inhibidores naturales de la enzima convertidora de angiotensina (ECA) provenientes de hidrolizados proteínicos de soya (Glycine max L. Merr.) (Moure et al., 2006) y frijol común (Hernández-Álvarez et al., 2008) o generados durante el procesamiento de granos (Wang y Gonzalez, 2005) podrían ser utilizados como fármacos o suplementos alimenticios para tratamiento de la hipertensión. Al ingerir la mezcla maíz+frijol se obtiene una mejora sustancial en el balance de aminoácidos, con calidad proteínica alta, minerales y vitaminas con nivel superior al obtenido si se consumieran por separado; además, por la suma de fitoquímicos, se incrementa el potencial nutracéutico.

La fermentación en estado sólido (FES) puede incrementar el contenido proteínico, digestibilidad proteínica y valor biológico de las proteínas, contenido de compuestos fenólicos, actividad antioxidante y potencial antihipertensivo (inhibición de enzima convertidora de angiotensina) y antidiabético (inhibición de actividades de a-amilasa y a-glucosidasa) en cereales y leguminosas (Bhanja et al., 2009; Lee et al., 2015; Sánchez-Magaña et al., 2014).

En México, harinas de maíz nixtamalizado, crudo, rostizado, germinado y fermentado, y harinas de arroz (Oryza sativa L.) y cebada (Hordeum vulgare L.) se utilizan para elaborar bebidas tradicionales (atole, tesgüino, pozol, horchata, cebada). Las bebidas funcionales son excelente medio para suministrar nutrimentos y compuestos bioactivos (péptidos, antioxidantes, vitaminas, minerales, ácidos grasos esenciales, fibra, prebióticos, probióticos). En 2016, la venta de alimentos y bebidas funcionales en el mundo rebasaría los 130 mil millones de dólares (Corbo et al., 2014).

El objetivo de esta investigación fue desarrollar una bebida funcional, con valor nutricional y potencial antihipertensivo y antidiabético alto, a partir de una mezcla 60:40 de harinas de granos integrales de maíz azul:frijol negro bioprocesados en condiciones optimizadas de fermentación en estado sólido (FES).

\section{MATERIALES Y MÉTODOS}

\section{Materiales biológicos}

Como materiales biológicos de estudio se utilizaron maíz criollo azul originario de la zona serrana de Sinaloa, sembrado en condiciones de riego en el Valle de Culiacán, y frijol negro var. Nayarit originario de Nayarit, recién cosechados.

\section{Proceso de fermentación en estado sólido (FES) para la obtención de harinas de maíz azul y frijol negro bioprocesados optimizadas (HMABO, HFNBO)}

Para la obtención de HMABO y HFNBO con actividad antioxidante (AAox) y contenido de compuestos fenólicos totales (CFT) altos, se aplicaron las metodologías propuestas por Cuevas-Rodríguez et al. (2006) y RochínMedina et al. (2015), respectivamente. Granos de maíz azul se fragmentaron en licuadora doméstica. Granos de maíz fragmentado y de frijol común, por separado, se remojaron en disolución de ácido acético ( $\mathrm{pH} 3.0$ ) a $25^{\circ} \mathrm{C}$ por 8 h. Las testas de los granos de frijol se removieron manualmente, se secaron, molturaron y almacenaron en bolsas de polietileno. Granos de maíz azul fragmentados y cotiledones de frijol acidificados se cocieron $\left(90^{\circ} \mathrm{C}\right.$ por $30 \mathrm{~min}$ ), drenaron, enfriaron $\left(25^{\circ} \mathrm{C}\right.$ por $3 \mathrm{~h}$ ), inocularon con Rhizopus oligosporus $\left(1 \times 10^{6}\right.$ esporas $\left.\mathrm{mL}^{-1}\right)$ y empacaron en bolsas de plástico $(15 \times 15 \mathrm{~cm})$ perforadas cada $\mathrm{cm}$. El maíz se incubó a $37{ }^{\circ} \mathrm{C}$ por $90 \mathrm{~h}$ y el frijol a $38^{\circ} \mathrm{C}$ por $100 \mathrm{~h}$. Los materiales bioprocesados se secaron $\left(50^{\circ} \mathrm{C}\right.$ por $8 \mathrm{~h}$ ), enfriaron $\left(23 \pm 2^{\circ} \mathrm{C}\right)$ y molturaron (malla 80$)$. La HMABO se empacó en bolsas de plástico. Los cotiledones de frijol bioprocesados se mezclaron con sus testas, previamente secadas y molturadas, para obtener HFNBO. Ambas harinas (HMABO y HFNBO) se almacenaron a $4{ }^{\circ} \mathrm{C}$ hasta su utilización.

\section{Extracción de compuestos fenólicos libres y ligados}

Los fenólicos libres en las harinas se extrajeron de acuerdo con Dewanto et al. (2002). A cada muestra (1.0 g) se le agregaron $10 \mathrm{~mL}$ de etanol-agua $(80: 20, v / v)$ y se 
colocó en agitador orbital (50 rpm por $10 \mathrm{~min}$ ). Las suspensiones se centrifugaron ( $3000 \mathrm{Xg}, 10 \mathrm{~min}$ ) (Sorvall ${ }^{\circledR} \mathrm{RC}$ 5 C, Sorvall Instruments, Dupont, Wilmington, DE, EUA) para recuperar sobrenadantes. Los extractos se concentraron a $2 \mathrm{~mL}$ a $45^{\circ} \mathrm{C}$ mediante un evaporador al vacío (Savant SC250 DDA Speed Vac Plus centrifugal, Holbrook, NY, EUA) y se almacenaron $\left(-20^{\circ} \mathrm{C}\right)$. Las extracciones se realizaron por triplicado.

Después de la extracción de fenólicos libres, el sedimento se resuspendió en $10 \mathrm{~mL}$ de $\mathrm{NaOH} 2 \mathrm{M}$. Las muestras se hidrolizaron a $95^{\circ} \mathrm{C}$ por 30 min y $25^{\circ} \mathrm{C}$ por 60 min en un baño de agua con agitación (60 rpm). El hidrolizado se neutralizó con $\mathrm{HCl}$ antes de la eliminación de lípidos con hexano. La suspensión final se extrajo cinco veces con $10 \mathrm{~mL}$ de acetato de etilo, el cual se evaporó a sequedad (Adom y Liu, 2002). Los fitoquímicos resultantes (fenólicos ligados) se resuspendieron en $2 \mathrm{~mL}$ de metanol al $50 \%$ y se almacenaron $\left(-20^{\circ} \mathrm{C}\right)$. Las extracciones se realizaron por triplicado.

\section{Actividad antioxidante (AAox)}

La AAox hidrofílica de extractos de fenólicos libres y ligados se evaluó mediante ensayo de capacidad de absorción de radicales oxígeno (ORAC, por sus siglas en inglés) con un lector de Microplacas (Synergy ${ }^{\top M}$ HT, BioTek, Inc., Winooski, VT, EUA). Los extractos se evaluaron frente a estándar de Trolox con fluoresceína (Ou et al., 2001). La AAox hidrofílica de fenólicos totales se calculó como la suma de AAox de fenólicos libres y ligados. La AAox se expresó en micromoles equivalentes de Trolox (ET) por $100 \mathrm{~g}$ de muestra (bs). Las mediciones se realizaron por triplicado.

\section{Compuestos fenólicos (CF) libres, ligados y totales}

El contenido de CF libres y ligados se evaluó con el método espectrofotométrico de Singleton et al. (1999). La absorbancia se midió mediante un lector de microplacas. El contenido de CF totales se calculó como la suma de CF libres y ligados. El contenido de CF se expresó como mg equivalentes de ácido gálico (EAG) / $100 \mathrm{~g}$ de muestra (bs). Las mediciones se realizaron por triplicado.

\section{Elaboración/evaluación sensorial de bebida funcional}

Cada porción de la bebida funcional $(200 \mathrm{~mL})$ se preparó con $25 \mathrm{~g}$ de mezcla ( $60 \%$ HMABO $+40 \%$ HFNBO), $170 \mathrm{~mL}$ de agua purificada, $1.5 \mathrm{~g}$ de SteviaMr y $1.5 \mathrm{~g}$ de vainilla (Vainilla) en polvo. Las bebidas se pasteurizaron, se envasaron en envases PET de $200 \mathrm{~mL}$ y se refrigeraron (de 4 a $6{ }^{\circ} \mathrm{C}$ ). La evaluación sensorial de las bebidas se realizó con 80 panelistas y se empleó una escala LAM (Labeled Affective Magnitude). Estos valores se transformaron a una escala de 0 a 100 (Cardello y Schutz, 2004).

\section{Composición proximal de la mezcla (60\% HMABO + $40 \%$ HFNBO)}

Métodos oficiales AOAC (1999) 925.09 B, 920.39 C, 960.52 y 923.03 se utilizaron para determinación de humedad, lípidos, proteína y cenizas; contenido de fibra y almidón resistente se determinaron según los métodos 985.29 y 2002.02, respectivamente. Las evaluaciones se hicieron por triplicado.

\section{Propiedades nutricionales de la mezcla $(60 \%$ HMABO $+40 \%$ HFNBO)}

Se determinó digestibilidad proteínica in vitro (DPIV) (Hsu et al., 1977), contenido de aminoácidos (López-Cervantes et al., 2006), calificación química (FAO, 2013) y relación de eficiencia proteínica calculada (C-PER) (Satterlee et al., 1982).

\section{Potencial antihipertensivo [Inhibición de la enzima convertidora de Angiotensina (ECA)] de las harinas bioprocesadas y su mezcla (60\% HMABO + $40 \%$ HFNBO)}

El potencial antihipertensivo se evaluó según el grado de inhibición de la enzima convertidora de angiotensina (ECA) por los hidrolizados proteínicos obtenidos con pancreatina a partir de las harinas por separado y mezcladas (HMA$\mathrm{BO}, \mathrm{HFNBO}, 60 \% \mathrm{HMABO}+40$ \% HFNBO) (Miguel et al., 2006). Este método se basa en la reacción desarrollada en una mezcla que contiene un amortiguador de borato de sodio $0.1 \mathrm{M}$, hipuril-histidil-leucina (HHL), ECA y diferentes diluciones de los hidrolizados proteínicos. La incubación se realizó a $37^{\circ} \mathrm{C}$ por $30 \mathrm{~min}$. La reacción se detuvo agregando $1 \mathrm{~mL}$ de acetato de etilo. Después de la reacción, la mezcla se centrifugó y se colectó la fase orgánica, donde se determinó la actividad enzimática. Se leyó la absorbancia a $228 \mathrm{~nm}$ con un lector de Microplacas (SynergyTM HT, BioTek, Inc., Winooski, VT, EUA). Se calculó entonces el valor $\mathrm{IC}_{50}$ [concentración del péptido en $\mu \mathrm{g}$ de proteína $/ \mathrm{mL}$ requerido para producir una inhibición del $50 \%$ de la actividad de ECA]; se determinó, por análisis de regresión, el \% de inhibición de ECA contra la concentración de péptidos. El IC 50 se calculó de la siguiente manera:

$\%$ Actividad inhibitoria $=\left[\left(\mathrm{Abs}_{\text {control }}-\mathrm{Abs}_{\text {muestra }}\right) /\left(\mathrm{Abs}_{\text {control }}-\mathrm{Abs}_{\text {blanco }}\right)\right] \times 100$

donde: $\mathrm{Abs}_{\text {control }}$ es la absorbancia del ácido hipúrico formado tras la acción de la ECA sin inhibidor, Abs $_{\text {blanco }}$ es la absorbancia del HHL que no reaccionó y que se extrajo con acetato de etilo, y $\mathrm{Abs}_{\text {muestra }}$ es la absorbancia del ácido hipúrico que se formó tras la acción de la ECA en presencia 
de sustancias inhibidoras.

\section{Potencial antidiabético [Índices de inhibición de a-amilasa (IIA) y a-glucosidasa (IIG)] de las harinas bioprocesadas y su mezcla}

La actividad inhibitoria de extractos de fenólicos libres y ligados de harinas contra a-amilasa se determinó por la medición colorimétrica de maltosa liberada después de detener la reacción entre almidón y a-amilasa con ácido 3,5-dinitrosalicílico (McCue et al., 2005). La actividad inhibitoria de estos extractos contra a-glucosidasa se determinó por la medición de la formación de p-nitrofenol por a-glucosidasa después de reaccionar con p-nitrofenil-aD-glucopiranósido (PNP) (Correia et al., 2004). Los IIA e IIG se definieron como la actividad de las enzimas en el control (enzima sola) / actividad de las enzimas con extracto. El valor de IIA para fenólicos totales se calculó como la suma de los valores de IIA en extractos de fenólicos libres y ligados. El mismo procedimiento se aplicó para determinar el valor de IIG de fenólicos totales. Valores de IIA e IIG > 1 indican inhibición enzimática.

\section{Análisis estadístico}

Los resultados experimentales se analizaron mediante un análisis de varianza de una sola vía y las medias se separaron aplicando la prueba de rango múltiple de Duncan, con un nivel de significancia del $5 \%$.

\section{RESULTADOS Y DISCUSIÓN}

\section{Efecto del bioprocesamiento sobre propiedades nutricionales de maíz azul y frijol negro}

En el Cuadro 1 se muestra el contenido de aminoácidos esenciales (AAE) en harinas de maíz azul y frijol negro crudos (sin procesar) y bioprocesados por fermentación en estado sólido (FES). La mayoría de los AAE de las proteínas de maíz azul y frijol negro incrementaron debido a la FES; solamente en el maíz azul el nivel de Val disminuyó $0.41 \mathrm{~g} / 100 \mathrm{~g}$ proteína, mientras que en el frijol negro el nivel de Lys disminuyó en $0.20 \mathrm{~g} / 100 \mathrm{~g}$ proteína. El efecto más significativo del bioprocesamiento por FES sobre las proteínas del maíz azul fue el incremento en Lys, lle y Trp. Estos aminoácidos incrementaron en 0.9, 0.4 y 0.08 g/100 g proteína, respectivamente.

Cuevas-Rodríguez et al. (2006) y Rochín-Medina et al. (2015) también reportaron incrementos en Lys, lle y Trp durante el bioprocesamiento de maíz QPM por FES. Por otro lado, las proteínas del frijol negro sufrieron pérdidas en Lys durante la FES; sin embargo, la disminución de Lys solamente representó un $3.08 \%$ del contenido de este AAE en el frijol negro, por lo que esta leguminosa es una buena fuente de este aminoácido esencial. Pérdidas de Lys durante el

Cuadro 1. Propiedades nutricionales de harinas de maíz azul y frijol negro.

\begin{tabular}{lccccc}
\hline Propiedad & $\begin{array}{c}\text { Maíz azul } \\
\text { crudo }\end{array}$ & HMABO & $\begin{array}{c}\text { Frijol negro } \\
\text { crudo }\end{array}$ & HFNBO & $\begin{array}{c}\text { Requerimientos de AAE } \\
\text { (3 años y mayores) }\end{array}$ \\
\hline Nutricional & & & & & \\
AAE (g/100 g proteína) & & & & & \\
His & $2.72 \pm 0.04 \mathrm{~b}$ & $3.42 \pm 0.04 \mathrm{a}$ & $2.41 \pm 0.02 \mathrm{c}$ & $2.59 \pm 0.03 \mathrm{bc}$ & 1.60 \\
Ile & $2.44 \pm 0.03 \mathrm{~d}$ & $2.86 \pm 0.02 \mathrm{c}$ & $3.08 \pm 0.04 \mathrm{~b}$ & $3.32 \pm 0.03 \mathrm{a}$ & 3.00 \\
Leu & $9.16 \pm 0.11 \mathrm{~b}$ & $10.54 \pm 0.12 \mathrm{a}$ & $7.20 \pm 0.06 \mathrm{c}$ & $7.19 \pm 0.06 \mathrm{c}$ & 6.10 \\
Lys & $2.72 \pm 0.06 \mathrm{~d}$ & $3.63 \pm 0.05 \mathrm{c}$ & $6.50 \pm 0.03 \mathrm{a}$ & $6.30 \pm 0.05 \mathrm{~b}$ & 4.80 \\
Met + Cys & $4.47 \pm 0.07 \mathrm{~b}$ & $4.87 \pm 0.05 \mathrm{a}$ & $2.14 \pm 0.03 \mathrm{~d}$ & $2.55 \pm 0.04 \mathrm{c}$ & 2.30 \\
Phe + Tyr & $6.92 \pm 0.10 \mathrm{~d}$ & $9.49 \pm 0.09 \mathrm{a}$ & $8.50 \pm 0.06 \mathrm{c}$ & $9.19 \pm 0.05 \mathrm{~b}$ & 4.10 \\
Thr & $3.51 \pm 0.05 \mathrm{c}$ & $4.33 \pm 0.05 \mathrm{a}$ & $3.50 \pm 0.04 \mathrm{c}$ & $3.75 \pm 0.02 \mathrm{~b}$ & 2.50 \\
Trp & $0.45 \pm 0.02 \mathrm{c}$ & $0.53 \pm 0.01 \mathrm{~b}$ & $1.36 \pm 0.02 \mathrm{a}$ & $1.29 \pm 0.02 \mathrm{a}$ & 0.66 \\
Val & $4.60 \pm 0.06 \mathrm{ab}$ & $4.19 \pm 0.04 \mathrm{c}$ & $4.51 \pm 0.02 \mathrm{~b}$ & $4.70 \pm 0.04 \mathrm{a}$ & 4.0 \\
Total & 36.99 & 43.86 & 39.20 & 40.88 & 29.06 \\
Calificación química & 56.7 & 75.6 & 93.0 & 100 & -- \\
Aminoácido limitante & Lys & Lys & Met + Cys & & \\
Dig proteínica in vitro (\%) & $83.20 \pm 0.12 \mathrm{~b}$ & $88.75 \pm 0.17 \mathrm{a}$ & $73.10 \pm 0.11 \mathrm{c}$ & $87.12 \pm 0.10 \mathrm{a}$ & \\
C-PER & $1.04 \pm 0.03 \mathrm{c}$ & $1.63 \pm 0.03 \mathrm{~b}$ & $1.58 \pm 0.05 \mathrm{~b}$ & $2.26 \pm 0.06 \mathrm{a}$ & \\
\hline
\end{tabular}

Medias con letras iguales en cada fila para cada propiedad no son estadísticamente diferentes (Duncan, P $\leq 0.05$ ); HMABO: harina de maíz azul bioprocesado optimizada; HFNBO: harina de frijol negro bioprocesado optimizada; AAE: aminoácidos esenciales; C-PER: relación de eficiencia proteínica calculada. 
bioprocesamiento por FES de frijol negro también fueron reportadas por Rochín-Medina et al. (2015). Ellos atribuyeron este decremento a la acción de las transaminasas producidas por el hongo Rhizopus oligosporus, las cuales son responsables de la conversión de aminoácidos; sin embargo, el hongo no emplea un aminoácido específico para su crecimiento, es por ello que el efecto sobre la composición de aminoácidos depende del sustrato utilizado.

El patrón sugerido por FAO (2013) para el requerimiento de aminoácidos esenciales para niños, adolescentes y adultos (3 años y mayores) fue empleado para evaluar la calificación química de las proteínas de las harinas estudiadas (Cuadro 1). En las proteínas de maíz azul sin procesar, el aminoácido esencial limitante fue Lys, con una calificación química de 56.7. La calificación química de AAE incrementó a 75.6 debido al bioprocesamiento por FES de este material. Los aminoácidos azufrados Met + Cys en las proteínas de frijol negro sin procesar fueron limitantes, con una calificación química de 93.0; la FES mejoró el balance de AAE en este material, que incrementó la calificación química de proteínas a 100, lo que significa que no existió AAE limitante en la harina de frijol negro bioprocesado.

La digestibilidad proteínica in vitro de maíz azul y frijol negro incrementó de 83.2 a $88.7 \%$ y de 73.1 a $87.1 \%$, respectivamente (Cuadro 1) durante el bioprocesamiento por FES. Estos resultados coinciden con los reportados por Rochín-Medina et al. (2015). La reducción de factores antinutricionales (ej. ácido fítico, inhibidores enzimáticos, taninos, etc.) por FES y la desnaturalización de proteínas durante la etapa de cocción incrementa la susceptibilidad de las proteínas a la hidrolisis enzimática, y como resultado incrementa la digestibilidad proteínica in vitro. La FES también incrementó la relación de eficiencia proteínica calculada (C-PER) de las muestras entre 43 y $57 \%$ (Cuadro 1). El incremento en C-PER puede ser atribuido al incremento en la digestibilidad proteínica in vitro y el contenido de AAE de las proteínas de los materiales estudiados.

\section{Efecto del bioprocesamiento sobre actividad antioxidante (AAox) y contenido de compuestos fenólicos totales (CFT) de maíz azul y frijol negro}

El bioprocesamiento de maíz azul y frijol negro mediante FES incrementó $(P \leq 0.05$ ) la AAox de 36.8 a 75.6 \% (Cuadro 2). La AAox de fitoquímicos libres y ligados incrementó (P $\leq 0.05$ ) de 234.5 a $163.4 \%$ y de 0 a $38 \%$, respectivamente, después de la FES (Cuadro 2). La FES incrementó ( $P \leq 0.05$ ) los contenidos de compuestos fenólicos libres, ligados y totales, de ambos sustratos, de 342.7 a $316.5 \%$, de 55.5 a $45.9 \%$, y de 85 a $127 \%$, respectivamente (Cuadro 2).

Los mayores incrementos de AAox y contenido de CFT

Cuadro 2. Contenido de compuestos fenólicos, actividad antioxidante y potenciales antihipertensivo y antidiabético de harinas de maíz azul y frijol negro.

\begin{tabular}{|c|c|c|c|c|}
\hline Propiedad & Maíz azul crudo & HMABO & Frijol negro crudo & HFNBO \\
\hline \multicolumn{5}{|c|}{ Compuestos fenólicos [mg equivalentes de ácido gálico (EAG)/100 g (bs)] } \\
\hline Libres & $29.18 \pm 0.37 d$ & $129.19 \pm 0.25 b$ & $57.23 \pm 0.34 c$ & $238.39 \pm 5.10 a$ \\
\hline Ligados & $248.53 \pm 3.65 b$ & $386.41 \pm 3.81 \mathrm{a}$ & $133.13 \pm 4.51 \mathrm{~d}$ & $194.21 \pm 4.60 \mathrm{c}$ \\
\hline Totales & $278.15 \pm 4.19 \mathrm{c}$ & $514.60 \pm 4.31 a$ & $190.67 \pm 4.27 \mathrm{~d}$ & $432.60 \pm 6.30 b$ \\
\hline \multicolumn{5}{|c|}{ Actividad antioxidante $[\mu \mathrm{mol}$ equivalentes de trolox $(\mathrm{ET}) / 100 \mathrm{~g}(\mathrm{bs})]$} \\
\hline Fenólicos libres & $2,906 \pm 72 d$ & $9,720 \pm 221 b$ & $3,884 \pm 130 \mathrm{c}$ & $10,230 \pm 213 a$ \\
\hline Fenólicos ligados & $14,887 \pm 304 a$ & $14,580 \pm 218 \mathrm{a}$ & $9,064 \pm 163 c$ & $12,503 \pm 254 b$ \\
\hline Fenólicos totales & $17,793 \pm 309 c$ & $24,300 \pm 331 \mathrm{a}$ & $12,948 \pm 225 d$ & $22,733 \pm 312 b$ \\
\hline Potencial antihipertensivo $\left(\mathrm{IC}_{50}\right)$ & ND & $358 \pm 11$ a & $79.22 \pm 0.21 b$ & $0.0145 \pm 0.008 c$ \\
\hline \multicolumn{5}{|l|}{ Índice de inhibición de a-amilasa } \\
\hline Fenólicos libres & $1.36 \pm 0.04 d$ & $3.18 \pm 0.07 b$ & $1.80 \pm 0.04 \mathrm{c}$ & $4.39 \pm 0.17 a$ \\
\hline Fenólicos ligados & $1.45 \pm 0.04 d$ & $2.35 \pm 0.07 b$ & $2.01 \pm 0.06 c$ & $2.52 \pm 0.06 a$ \\
\hline Fenólicos totales & $2.81 \pm 0.09 d$ & $5.53 \pm 0.14 b$ & $3.81 \pm 0.15 c$ & $6.91 \pm 0.20 a$ \\
\hline \multicolumn{5}{|c|}{ Índice de inhibición de a-glucosidasa } \\
\hline Fenólicos libres & $1.19 \pm 0.04 \mathrm{c}$ & $1.38 \pm 0.03 b$ & $1.26 \pm 0.04 c$ & $1.69 \pm 0.05 a$ \\
\hline Fenólicos ligados & $1.42 \pm 0.05 d$ & $2.99 \pm 0.08 b$ & $1.94 \pm 0.04 \mathrm{c}$ & $3.85 \pm 0.11 \mathrm{a}$ \\
\hline Fenólicos totales & $2.61 \pm 0.09 d$ & $4.37 \pm 0.13 b$ & $3.20 \pm 0.09 c$ & $5.54 \pm 0.12 a$ \\
\hline
\end{tabular}

Medias con letras iguales en cada fila y para cada propiedad no son estadísticamente diferentes (Duncan, P $\leq 0.05$ ); HMABO: harina de maíz azul bioprocesado optimizada; HFNBO: harina de frijol negro bioprocesado optimizada; $\mathrm{IC}_{50}: \mathrm{\mu g} \mathrm{mL}^{-1}$ de suspensión; ND: no determinado. 
se observaron en frijol. Estos resultados coinciden con lo reportado por otros investigadores (Cheng et al., 2013; Salar et al., 2012; Xiao et al., 2016) quienes encontraron que la FES incrementó el contenido de compuestos fenólicos de cereales y leguminosas bioprocesadas, lo cual mejora su funcionalidad y potencial terapéutico. La mayoría de los compuestos fenólicos en plantas se encuentran como glucósidos, conjugados con azúcares. Se ha sugerido (Lin et al., 2006; Maiti y Majumdar, 2012) que la enzima fúngica $\beta$-glucosidasa cataliza la liberación de agliconas del sustrato $y$, como consecuencia, se observa un incremento en contenido de compuestos fenólicos. Salar et al. (2012) confirmaron la participación de algunas enzimas ( $a$-amilasa, $\beta$-glucosidasa, xilanasa) en la movilización de compuestos fenólicos en maíz durante la FES de este cereal con Thamnidium elegans.

\section{Efecto del bioprocesamiento sobre potencial antihipertensivo [Actividad inhibitoria de la enzima convertidora de Angiotensina (ECA)] de maíz azul y frijol negro}

La HMABO tuvo un $\mathrm{IC}_{50}$ de $358 \mu \mathrm{g} \mathrm{mL} \mathrm{L}^{-1}$ pero no se determinó el valor respectivo en maíz crudo; por su parte, la HFNBO tuvo mejor $\mathrm{IC}_{50}$ que el frijol negro crudo (0.01 vs $79.2 \mu \mathrm{g} \mathrm{mL}^{-1}$ ) (Cuadro 2). Los resultados obtenidos son alentadores: los valores de $\mathrm{IC}_{50}$ están en concordancia o son menores que los reportados para hidrolizados proteínicos de frijol común crudo (por medio de pancreatina) (60 a $319 \mathrm{mg} \mathrm{mL}^{-1}$ ) (Valdez-Ortiz et al., 2012) y de frijol lima crudo (Phaseolus lunatus) (mediante pepsina + pancreatina) (250 a $692 \mu \mathrm{g} \mathrm{mL}^{-1}$ ) (Chel-Guerrero et al., 2012).

Durante el bioprocesamiento de maíz o frijol común a través de FES se produce la liberación de fitoquímicos con actividad biológica e hidrólisis parcial de proteínas, lo cual se suma a la digestión gastrointestinal, donde se liberan diversos péptidos y muchos otros compuestos bioactivos con capacidad de inhibición de la enzima convertidora de angiotensina (ECA); con ello se reduce la actividad de angiotensina II e incrementan los niveles de bradiquidina, lo que resulta en una disminución de la presión arterial. Se ha reportado el aislamiento de inhibidores naturales de ECA a partir de la hidrólisis de proteínas de varios granos como amaranto (Amaranthus hypochondriacus L.) (Silva-Sánchez et al., 2008) y frijol común (Valdez-Ortiz et al., 2012) que podrían ser utilizados como fármacos o suplementos alimenticios funcionales para el tratamiento de la hipertensión.

\section{Efecto del bioprocesamiento sobre potencial antidiabético [índices de inhibición de a-amilasa (IIA) y a-glucosidasa (IIG)] de maíz azul y frijol negro}

La enzima a-amilasa es responsable de la hidrólisis de almidón durante el proceso digestivo, lo cual es importante para manejo postprandial de niveles de glucosa en sangre. Todas las muestras analizadas tuvieron valores de IIA > 1.0, lo que indica que poseen actividad antiamilasa (Cuadro 2). La FES incrementó $(P \leq 0.05)$ el IIA de fitoquímicos libres, ligados y totales de maíz azul y frijol negro. Este incremento en el IIA, atribuido a CFT, para maíz azul y frijol negro fue de $96.8 \%$ y $81.4 \%$, respectivamente (Cuadro 2 ).

La FES incrementó $(P \leq 0.05)$ el IIG de fitoquímicos libres, ligados y totales de maíz azul y frijol negro. Este incremento en el IIG, atribuido a CFT, para maíz azul y frijol negro fue de $67.4 \%$ y $73.1 \%$, respectivamente (Cuadro 2). Valores de $I I G>1.0$ indican que los extractos evaluados poseen actividad antiglucosidasa.

Maiti y Majumdar (2012) reportaron que extractos de soya bioprocesada con $R$. oligosporus tuvieron IIA $=3.6$ al sexto día de incubación. Sánchez-Magaña et al. (2014) observaron, en extractos de garbanzo (Cicer arietinum) bioprocesado $\left(35^{\circ} \mathrm{C}\right.$ por $108 \mathrm{~h}$ ) con $R$. oligosporus IIA = 5.77; además, observaron una correlación altamente significativa entre CFT e IIA.

Una importante alternativa terapéutica para el manejo de las primeras etapas de la diabetes tipo 2 es el control de la absorción de glucosa a través de la reducción de la hidrólisis del almidón mediante la inhibición de las enzimas a-amilasa y a-glucosidasa. Estudios clínicos en humanos utilizando inhibidores naturales de a-amilasa y a-glucosidasa provenientes de plantas alimenticias (e.g. frijol común blanco) han demostrado excelentes efectos antihiperglicémicos sin causar efectos colaterales como diarrea, distinción intestinal, vómito y flatulencia, los cuales son comúnmente encontrados con el uso de medicamentos disponibles comercialmente, promocionados como inhibidores terapéuticos de a-amilasa y a-glucosidasa (Barrett y Udani, 2011; Fujita et al., 2001, 2003).

Inhibidores de a-amilasa y a-glucosidasa "grado alimenticio" provenientes de extractos de HMABO y HFNBO son potencialmente seguros y podrían ser utilizados como alternativa para la modulación de la digestión de carbohidratos y el control del índice glicémico de productos alimenticios. Incrementos en los IIA e IIG en extractos de maíz azul y frijol negro, como consecuencia de la FES, no se han reportado previamente en la literatura. 


\section{Composición nutrimental, contenido de energía y propiedades nutricionales de la bebida funcional}

Una porción de $200 \mathrm{~mL}$ de la bebida preparada con 25 $\mathrm{g}$ de la mezcla funcional (60 \% HMABO + $40 \% \mathrm{HFNBO}$ ) contiene $4.4 \mathrm{~g}$ de proteína, $0.5 \mathrm{~g}$ de lípidos, $16.7 \mathrm{~g}$ de carbohidratos y $90.2 \mathrm{kcal}$. Esta porción de la bebida funcional cubre el $34.2 \%$ y el $23.4 \%$ de los requerimientos diarios de proteína para niños de 1 a 3 y de 4 a 8 años de edad, respectivamente. Esta bebida funcional también contiene $5.55 \mathrm{~g}$ de fibra dietaria total ( $1.7 \mathrm{~g}$ de fibra soluble y $3.8 \mathrm{~g}$ de fibra insoluble) y $0.8 \mathrm{~g}$ de almidón resistente. La fibra dietaria y el almidón resistente en alimentos son considerados ingredientes funcionales para el combate a la obesidad y la reducción de la incidencia de cáncer de colon (Prado-Silva et al., 2014).

El contenido de aminoácidos esenciales (AAE) en la bebida funcional fue mayor que el recomendado (FAO, 2013) para niños mayores, adolescentes y adultos ( 3 años y mayores) (42.0 vs 29.0 g/100 g proteína), su calificación química fue de 100 y no presentó AAE limitante (Cuadro 3).
La bebida funcional tuvo digestibilidad proteínica in vitro (DPIV) de 88.1 \% y relación de eficiencia proteínica calculada (C-PER) de 2.3 (Cuadro 3).

Las excelentes propiedades nutricionales de la bebida funcional elaborada a partir de la mezcla $60 \%$ HMABO + $40 \%$ HFNBO se deben esencialmente a la mejora que sufrió la calidad de la proteína de los granos de maíz azul y frijol negro durante el bioprocesamiento por FES. Otros investigadores han reportado mejora nutricional de la proteína durante la FES de diferentes granos procesados; por ejemplo Niveditha y Sridhar (2016) Ilevaron a cabo fermentación de granos de Canavalia cathartica con Rhizopus oligosporus y reportaron, por efecto de la FES, incrementos en el contenido de proteína verdadera y en el aminoácido esencial Lys, al igual que en la digestibilidad proteínica in vitro. Ellos reportaron una relación de eficiencia proteínica (PER, por sus siglas en inglés) de 0.7 para granos cocidos y 1.1 para granos fermentados.

En cuanto al resultado de la evaluación sensorial de la bebida funcional, los panelistas evaluaron con un valor

Cuadro 3. Propiedades nutricionales y nutracéuticas de una porción de $200 \mathrm{~mL}$ de bebida funcional preparada con $25 \mathrm{~g}$ de la mezcla funcional (60 \% HMABO + $40 \%$ HFNBO).

\begin{tabular}{|c|c|c|c|}
\hline \multirow{2}{*}{$\begin{array}{l}\text { Propiedad } \\
\text { Nutricional }\end{array}$} & \multicolumn{2}{|c|}{ Bebida funcional } & \multirow[t]{2}{*}{$\begin{array}{c}\text { Requerimientos de AAE } \\
\text { (3 años y mayores) }\end{array}$} \\
\hline & & & \\
\hline Aminoácido esencial & (g) & (g/100 g proteína) & (g/100 g proteína) \\
\hline His & 0.13 & $2.91 \pm 0.03$ & 1.60 \\
\hline Ile & 0.14 & $3.14 \pm 0.03$ & 3.00 \\
\hline Leu & 0.38 & $8.51 \pm 0.05$ & 6.10 \\
\hline Lys & 0.23 & $5.25 \pm 0.06$ & 4.80 \\
\hline Met+Cys & 0.15 & $3.46 \pm 0.03$ & 2.30 \\
\hline Phe+Tyr & 0.41 & $9.31 \pm 0.10$ & 4.10 \\
\hline Thr & 0.18 & $3.98 \pm 0.07$ & 2.50 \\
\hline Trp & 0.04 & $0.99 \pm 0.02$ & 0.66 \\
\hline Val & 0.20 & $4.50 \pm 0.04$ & 4.00 \\
\hline Total & 1.87 & 42.06 & 29.06 \\
\hline Calificación química & --- & 100 & --- \\
\hline Aminoácido limitante & --- & --- & --- \\
\hline Digestibilidad proteínica in vitro (\%) & \multicolumn{2}{|c|}{$88.1 \pm 0.22$} & --- \\
\hline C-PER & \multicolumn{2}{|c|}{$2.39 \pm 0.04$} & --- \\
\hline \multicolumn{4}{|l|}{ Nutracéutica } \\
\hline Actividad antioxidante & \multicolumn{2}{|c|}{$4,500 \pm 357$} & --- \\
\hline Potencial antihipertensivo $\left(\mathrm{IC}_{50}\right)$ & \multicolumn{2}{|c|}{$25.12 \pm 0.53$} & --- \\
\hline Índice de inhibición de a-amilasa & \multicolumn{2}{|c|}{$6.16 \pm 0.12$} & --- \\
\hline Índice de inhibición de a-glucosidasa & \multicolumn{2}{|c|}{$4.87 \pm 0.17$} & --- \\
\hline
\end{tabular}

HMABO: harina de maíz azul bioprocesado optimizada; HFNBO: harina de frijol negro bioprocesado optimizada; C-PER: relación de eficiencia proteínica calculada; actividad antioxidante: $\mu \mathrm{mol}$ equivalentes de Trolox; $\mathrm{IC}_{50}: \mu \mathrm{g} \mathrm{mL}^{-1}$ suspensión. 
promedio de 82 la aceptabilidad de la bebida (entre "me gusta mucho" y "me gusta extremadamente").

Existen pocas opciones de bebidas en el mercado que ofrezcan un buen contenido de proteína de buena calidad, fibra dietaria, almidón resistente y un contenido energético menor a 100 kcal por porción.

\section{Propiedades nutracéuticas de la bebida funcional}

La bebida funcional $(200 \mathrm{~mL})$ preparada con la mezcla funcional $60 \%$ HMABO + $40 \%$ HFNBO tuvo una AAox de $4500 \mu \mathrm{mol}$ ET, y contribuye con 90 a $150 \%$ del consumo diario recomendado de antioxidantes (3000 a 5000 ORAC; ACES, 2010). Esta bebida también tuvo un potencial antihipertensivo, expresado como $\mathrm{IC}_{50^{\prime}}$ de $25.12 \mu \mathrm{g} \mathrm{mL} \mathrm{m}^{-1}$ de suspensión (Cuadro 3). Este valor antihipertensivo $\left(\mathrm{IC}_{50}\right)$ de la bebida funcional fue proporcionado básicamente por la mezcla funcional $60 \% \mathrm{HMABO}+40 \%$ HFNBO. Existen otros reportes en la literatura de $\mathrm{IC}_{50}$ para otras harinas funcionales. Torino et al. (2013) reportaron un valor de $I C_{50}$ para lenteja (Lens culinaris L.) fermentada de 180 a 200 $\mu \mathrm{g} \mathrm{mL}$.

El potencial antidiabético de la bebida funcional, expresado a través de valores de IIA e IIG, los cuales deben ser mayores a la unidad para garantizar efectos antiamilasa y antiglucosidasa, respectivamente, fue de $\| \mathrm{A}=6.16$ e $\| \mathrm{G}=$ 4.87 (Cuadro 3). Estos valores de inhibición de las enzimas encargadas de la digestión de carbohidratos en seres humanos por la bebida funcional son muy altos, por lo que tienen potencial para contribuir en el control de la diabetes y el sobrepeso u obesidad en personas que tienen estos padecimientos.

Esta bebida, debido a que presenta valor nutricional y potencial antioxidante, antihipertensivo y antidiabético alto, puede ser considerada una bebida funcional, y por su aceptabilidad sensorial alta representa una excelente alternativa para promover su consumo y la salud de los consumidores.

\section{CONCLUSIONES}

La FES es una estrategia biotecnológica que incrementó el valor nutricional, la actividad antioxidante (AAox), el contenido de compuestos fenólicos totales (CFT) y los potenciales antihipertensivo y antidiabético de maíz azul y frijol negro. La bebida funcional preparada con una mezcla de harinas de maíz azul y frijol negro bioprocesados por FES presentó un contenido alto de proteínas de buena calidad, fibra dietaria y almidón resistente, con un contenido menor a $100 \mathrm{kcal}$ de energía y una elevada aceptabilidad sensorial. Asimismo, la bebida funcional presentó un potencial antioxidante, antihi- pertensivo y antidiabético elevado. Es por ello que, esta bebida funcional por su valor nutricional y potencial nutracéutico, puede ser empleada para la promoción de la salud y como una alternativa a las bebidas de contenido calórico alto y valor nutricional que cuales predominan en el mercado.

\section{AGRADECIMIENTOS}

Esta investigación recibió financiamiento del Programa de Fomento y Apoyo a Proyectos de Investigación (PROFAPI) 2013, 2014 de la Universidad Autónoma de Sinaloa.

\section{BIBLIOGRAFÍA}

ACES, Alabama Cooperative Extension System (2010) Antioxidants and heatlh. Alabama Cooperative Extension System Publication HE-778. Betsville. $4 \mathrm{p}$.

AOAC (1999) Official Methods of Analysis. $16^{\circ}$ ed. Association of Official Analytical Chemists. St. Paul, MN, USA.

Adom K. K. and R. H. Liu (2002) Antioxidant activity of grains. Journal of Agricultural and Food Chemistry 50:6182-6187.

Avendaño A. C. H., P. Ramírez V., F. Castillo G., J. L. Chávez S. y G. Rincón E. (2004) Diversidad isoenzimática en poblaciones nativas de frijol negro. Revista Fitotecnia Mexicana 27:31-40.

Barrett M. L. and J. K. Udani (2011) A proprietary alpha-amylase inhibitor from white bean (Phaseolus vulgaris): a review of clinical studies on weight loss and glycemic control. Nutrition Journal 10:24.

Bhanja T., A. Kumari and R. Banerjee (2009) Enrichment of phenolics and free radical scavenging property of wheat koji prepared with two filamentous fungi. Bioresource Technology 100:2861-2866.

Cardello A. V. and H. G. Schutz (2004) Numerical scale-point locations for constructing the LAM (labeled affective magnitude) scale. Journal of Sensory Studies 19: 341-346.

Chávez-Santoscoy R. A., J. A. Gutiérrez-Uribe, S. 0. Serna-Saldivar and E. Pérez-Carrillo (2016) Production of maize tortillas and cookies from nixtamalized flour enriched with anthocyanins, flavonoids and saponins extracted from black bean (Phaseolus vulgaris) seed coats. Food Chemistry 192:90-97.

Chel-Guerrero L., M. Domínguez-Magaña, A. Martínez-Ayala. G. Dávila-Ortiz and D. Betancur-Ancona (2012) Lima bean (Phaseolus lunatus) protein hydrolysates with ACE-I inhibitory activity. Food and Nutrition Sciences 3:511-521.

Cheng K. C., J. Y. Wu, J. T. Lin and W. H. Liu (2013) Enhancement of isoflavones aglycones, total phenolic content, and antioxidant activity of black soybean by solid-state fermentation with Rhizopus spp. European Food Research and Technology 236:1107-1113.

Corbo M. R., A. Bevilacqua, L. Petruzzi, F. P. Casanova and M. Sinigaglia (2014) Functional beverages: the emerging side of functional foods. Commercial trends, research, and health implications. Comprehensive Reviews in Food Science and Food Safety 13:11921206.

Correia R. T. P., P. McCue, D. A. Vattem, M. M. A. Magalhães, G. R. Macêdo and K. Shetty (2004) Amylase and Helicobacter pylori inhibition by phenolic extracts of pineapple wastes bioprocessed by Rhizopus oligosporus. Journal of Food Biochemistry 28:419-434.

Cuevas-Rodríguez E. O., N. M. Verdugo-Montoya, P. I. Angulo-Bejarano, J. Milán-Carrillo, R. Mora-Escobedo, L. A. Bello-Pérez, J. A. Garzón-Tiznado and C. Reyes-Moreno (2006) Nutritional properties of tempeh flour from quality protein maize (Zea mays L). LWT - Food Science and Technology 39:1072-1079.

Dewanto V., X. Wu and R. H. Liu (2002) Processed sweet corn has higher antioxidant activity. Journal of Agricultural and Food Chemistry 50:4959-4964.

FAO, Food and Agriculture Organization (2013) Findings and recommendations of the 2011 FAO expert consultation on protein quality evaluation in human nutrition. In: Dietary Protein Quality Evaluation in Human Nutrition: Report of an FAO Expert Consultation. FAO Food and Nutrition Paper 92. Food and Agriculture Organization of the United Nations. Rome, Italy. pp:19-50. 
Fernández S. R., L. A. Morales C. and A. Gálvez M. (2013) Importancia de los maíces nativos de México en la dieta nacional. Una revisión indispensable. Revista Fitotecnia Mexicana 36:275-283.

Fujita H., T. Yamagami and K. Ohshima (2001) Efficacy and safety of Touchi extract, an a-glucosidase inhibitor derived from fermented soybeans, in non-insulin-dependent diabetic mellitus. The Journal of Nutritional Biochemistry 12:351-356.

Fujita H., T. Yamagami and K. Ohshima (2003) Long-term ingestion of Touchi-extract, an a-glucosidase inhibitor, by borderline and mild type-2 diabetic subjects is safe and significantly reduces blood glucose levels. Nutrition Research 23:713-722

Hernández-Álvarez A. J., G. Dávila-Ortız, C. Jiménez-Martínez and C. Jacinto-Hernández (2008) Isolation and characterization of bioactive peptides from seed of bean (Phaseolus vulgaris var. Jamapa) Food Science and Food Biotechnology in Developing Countries 19:505-509.

Hsu H. W., D. L. Vavak, L. D. Satterlee and G. A. Miller (1977) A multienzyme technique for estimating protein digestibility. Journal of Food Science 42:1269-1273.

Kim Y. M., Y. K. Jeong, M. H. Wang, W. Y. Lee and H. I. Rhee (2005) Inhibitory effect of pine extract on a-glucosidase activity and postprandial hyperglycemia. Nutrition 21:756-761.

Lee B. H., Y. S. Lai and S. C. Wu (2015) Antioxidation, angiotensin converting enzyme inhibition activity, nattokinase, and antihypertension of Bacillus subtilis (natto)-fermented pigeon pea. Journal of Food and Drug Analysis 23:750-757.

Lin C. H., Y. T. Wei and C. C. Chou (2006) Enhanced antioxidative activity of soybean koji prepared with various filamentous fungi. Food Microbiology 23:628-633.

López-Cervantes J., D. I. Sánchez-Machado and J. A. Rosas-Rodríguez (2006) Analysis of free amino acids in fermented shrimp waste by high-performance liquid chromatography. Journal of Chromatography A 1105:106-110.

Maiti D. and M. Majumdar (2012) Impact of bioprocessing on phenolic content and antioxidant activity of two edible seeds to improve hypoglycemic functionality. Journal of Natural Pharmaceuticals 3:31-36

McCue P., Y. I. Kwon and K. Shetty (2005) Anti-diabetic and anti-hypertensive potential of sprouted and solid-state bioprocessed soybean. Asia Pacific Journal of Clinical Nutrition 14:145-152.

Miguel M., M. A. Aleixandre, M. Ramos and R. López-Fandiño (2006) Effect of simulated gastrointestinal digestion on the antihypertensive properties of ACE-inhibitory peptides derived from ovalbumin Journal of Agricultural and Food Chemistry 54:726-731.

Moure A., H. Domínguez and J. C. Parajó (2006) Antioxidant properties and ultrafiltration-recovered soy protein fractions from industrial effluents and their hydrolysates. Process Biochemistry 41:447456.

Niveditha V. R. and K. R. Sridhar (2016) Improvement of nutritional qualities of fermented kernels of wild legume Canavalia cathartica by Rhizopus oligosporus. Current Biochemical Engineering 3:128-138.

Ou B., M. Hampsch-Woodill and R. L. Prior (2001) Development and validation of an improved oxygen radical absorbance capacity assay using fluorescein as the fluorescent probe. Journal of Agricultural and Food Chemistry 49:4619-4626.

Prado-Silva L., L. Azevedo, J. A. C. Oliveira, A. P. M. Moreira, M. Schmiele, Y. K. Chang, F. B. A. Paula and M. T. P. S. Clerici (2014) Sesame and resistant starch reduce the colon carcinogenesis and oxidative stress in 1,2-dimethylhydrazine-induced cancer in Wistar rats. Food Research International 62:609-617.

Rochín-Medina J. J., R. Gutiérrez-Dorado, L. M. Sánchez-Magaña, J. MilánCarrillo, E. O. Cuevas-Rodríguez, S. Mora-Rochín, A. Valdez-Ortiz and C. Reyes-Moreno (2015) Enhancement of nutritional properties, and antioxidant and antihypertensive potential of black common bean seeds by optimizing the solid state bioconversion process. International Journal of Food Sciences and Nutrition 66:498-504

Salar R. K., M. Certik and V. Brezova (2012) Modulation of phenolic content and antioxidant activity of maize by solid state fermentation with Thamnidium elegans CCF 1456. Biotechnology and Bioprocess Engineering 17:109-116.

Sánchez G. J. J., M. M. Goodman and C. W. Stuber (2000) Isozymatic and morphological diversity in the races of maize of Mexico. Economic Botany 54:43-59.

Sánchez-Magaña L. M., E. O. Cuevas-Rodríguez, R. Gutiérrez-Dorado, A. E. Ayala-Rodríguez, A. Valdez-Ortiz, J. Milán-Carrillo and C. ReyesMoreno (2014) Solid-state bioconversion of chickpea (Cicer arietinum L.) by Rhizopus oligosporus to improve total phenolic content, antioxidant activity and hypoglycemic functionality. International Journal of Food Sciences and Nutrition 65:558-564.

Satterlee L. D., J. G. Kendrick, H. F. Marshall, D. K. Jewell, R. A. Ali, M. M. Heckman, H. F. Steinke, P. Larson, R. D. Phillips, G. Sarwar and P. Slump (1982) In vitro assay for predicting protein efficiency ratio as measured by rat bioassay: collaborative study. Journal - Association of Analytical Chemists 65:798-809.

Serna-Saldívar S. O., J. A. Gutiérrez-Uribe, S. Mora-Rochín y S. García-Lara (2013) Potencial nutracéutico de los maíces criollos y cambios durante el procesamiento tradicional y con extrusión. Revista Fitotecnia Mexicana 36:295-304

Silva-Sánchez C., A. P. Barba R., M. F. León-Galván, B. O. de Lumen, A. de León-Rodríguez and E. González de M. (2008) Bioactive peptides in amaranth (Amaranthus hypochondriacus) seed. Journal of Agricultural and Food Chemistry 56:1233-1240.

Singleton V. L., R. Orthofer and R. M. Lamuela-Raventós (1999) Analysis of total phenols and other oxidation substrates and antioxidants by means of folin-ciocalteu reagent. Methods in Enzymology 299:152-178

Torino M. I., R. I. Limón, C. Martínez-Villaluenga, S. Mäkinen, A. Pihlanto, C. Vidal-Valverde and J. Frias (2013) Antioxidant and antihypertensive properties of liquid and solid state fermented lentils. Food Chemistry 136:1030-1037.

Valdez-Ortiz A., C. I. Fuentes-Gutiérrez, L. J. Germán-Báez, R. Gutiérrez-Dorado and S. Medina-Godoy (2012) Protein hydrolysates obtained from Azufrado (sulphur yellow) beans (Phaseolus vulgaris): nutritional, ACE-inhibitory and antioxidative characterization. LWT - Food Science and Technology 46:91-96.

Wang W. and E. Gonzalez de M. (2005) A new frontier in soy bioactive peptides that may prevent age-related chronic diseases. Comprehensive Reviews in Food Science and Food Safety 4:63-78.

Xiao Y., J. Fan, Y. Chen, X. Rui, Q. Zhang and M. Dong (2016) Enhanced total phenolic and isoflavone aglycone content, antioxidant activity and DNA damage protection of soybeans processed by solid state fermentation with Rhizopus oligosporus RT-3. RSC Advances 6:29741-29756. 
